Volume 9, No.4, July - August 2020

International Journal of Advanced Trends in Computer Science and Engineering

Available Online at http://www.warse.org/IJATCSE/static/pdf/file/ijatcse151942020.pdf

https://doi.org/10.30534/ijatcse/2020/151942020

\title{
A New Algorithm for Baseband Pulse Transmission over Band-Limited Channels for Wireless Automotive Communications
}

\author{
Ahmed F. Ashour ${ }^{1}$, Ashraf Ramadan ${ }^{2}$, Aziza I. Hussein ${ }^{3}$, Ashraf A. M. Khalaf ${ }^{4}$, Hesham F. A. Hamed \\ ${ }^{1,2}$ Higher Technological Institute, Electrical and Computer Engineering Department, Tenth of Ramadan, Egypt \\ 1'ahmed.ashour@hti.edu.eg, ${ }^{2}$ drashraf.ramadan6@gmail.com, ${ }^{3}$ azibrahim@effatuniversity.edu.sa \\ ${ }^{4}$ ashkhalaf@yahoo.com, ${ }^{5}$ hesham.fathi@mu.edu.eg \\ ${ }^{3}$ Effat University, Electrical and Computer Engineering Department, Jeddah, KSA \\ ${ }^{4}$ Minia University, Faculty of Engineering, Department of Electronics and Communications Eng., Minia, Egypt \\ ${ }^{5}$ Egyptian Russian University, Department of Telecommunications Eng., Cairo, Egypt
}

\begin{abstract}
A new technique for sending a baseband pulse signal from its band-pass spectral information is presented. It is advantageous if the pulse low-pass spectral information is not available or even if only a band-pass window of the pulse spectral information is available. This will help in sending any baseband pulse over a band limited channel. The new technique is expected to be very efficient compared to other wellknown pulse transmission techniques for which the major part of the signal spectral power is unavailable. The presented technique utilizes the pulse signal available spectral power provided by the available band-pass spectrum which must be measured rather accurately. A new recovery algorithm has been used for reconstructing the baseband pulse signal. This algorithm is run for different segments and different iterations and showed a good performance for recovering more than $90 \%$ of signal's energy. The new technique is implemented on a MATLAB platform. It has been applied for a base band pulse signal and should find applications in highdata-rate vehicle to vehicle wireless communications.
\end{abstract}

Keywords: Pulse Transmission, Band limited channel, Wireless Automotive communications, Vehicle-to-vehicle communications.

\section{INTRODUCTION}

A classical tool for characterizing the frequency components of a signal $f(t)$ whose domain is the finite-extent time inter$\operatorname{val}(-\tau / 2, \tau / 2)$ is that of Fourier analysis. In particular, such an analysis is begun by first evaluating the Fourier Transform (FT) integral [1]

$$
F(\omega)=\int_{-\infty}^{\infty} f(t) e^{-j \omega t} d t
$$

In which $\omega$ is taken to be a real frequency variable with values in the range $(-\infty, \infty)$. The Fourier spectrum associated with signal $f(t)$ is then defined to be the magnitude quantity $|F(\omega)|$ and its behavior as a function of $\omega$ often yields useful information relative to the signal $f(t)$.
Conceptually, the evaluation of this transform integral is straightforward, and its utility is well known.

In any real-world application, however, it must be realized that only a finite frequency observation of the signal to be inverse transformed is ever available (e.g. due to measuring devices limitations) which means that the signal under consideration has a spectrum which is known to fall within a given frequency band as specified by the set

$$
\Omega=\left\{\omega: \omega_{s} \leq|\omega| \leq \omega_{e}\right\}
$$

where $\omega_{s}$ and $\omega_{e}$ are positive frequency starting and end of measurement respectively which identify the known (measured) band pass interval of the signal.

Clearly, since only the partial spectral behaviour of the signal $f(t)$ isspecified by

$$
F(\omega) \text { for } \omega_{s} \leq|\omega| \leq \omega_{e}
$$

is given, one is unable to evaluate the Inverse Fourier Transform (IFT) [1]

$$
f(t)=\frac{1}{2 \pi} \int_{-\infty}^{\infty} F(\omega) e^{j \omega t} d \omega
$$

In such practical situations, it is then necessary to find suitably good estimates of the IFT based on this partial information. In this paper, we shall be concerned with developing a new technique for extrapolating a finite- frequency segment of a time-limited baseband pulse signal.

This will help to send a baseband pulse signal over any bandlimited channel. The basis of such a technique is the fact that if the signal spectrum $F(\omega)$ is known within an arbitrarily small neighbourhood centred at $\omega=\omega_{0}$, then we can compute the value of the function and all its derivatives at $\omega_{0}$, and generate a Taylor series about $\omega=\omega_{0}$ which can be used for the extrapolation [2].

$$
F(\omega)=\frac{\sum_{n=-\infty}^{\infty}\left(\omega-\omega_{o}\right)^{n} F^{(n)}(\omega)}{n !}
$$


Ahmed F. Ashour et al., International Journal of Advanced Trends in Computer Science and Engineering, 9(4), July - August 2020, 5222 - 5228

where $F^{(n)}\left(\omega_{o}\right)$ is the $\mathrm{n}^{\text {th }}$ derivative of $F(\omega)$ at $\omega=\omega_{o}$. In practice this can be done to an approximation. We could, for example, empirically determine $F^{(0)}\left(\omega_{o}\right)$ and $F^{(1)}\left(\omega_{o}\right)$ and maybe even $F^{(2)}\left(\omega_{o}\right)$. But the higher order derivative determination will become critically muddled by measurement in exactitude.

Thus, at best case, one could best fit a quadratic to the signal at $\omega=\omega_{o}$, so it is clear that this procedure is not practical and subject to an extremely unpredicted error due to approximations. Time-domain signal reconstruction algorithms and schemes for different applications have been discussed in [3] $-[7]$.

Pulse signal recovery has been widely explored in different research fields including optical, X-ray, image processing, antenna, radar, magnetic and biological systems [8] - [11]. Practically, extrapolation has been a good solution for a variety of low-pass reconstruction problems [12] - [14].

The new technique presented in this contribution extends the extrapolation techniques and applies it to the case of baseband pulse transmission to be transmitted over any bandlimited channel. In the following sections, our discussions will be based on the assumptions that it is required to send the baseband pulse signal over a noiseless channel.

This is for illustrating the new baseband pulse transmission concept and in further coming papers, we will consider the case of noisy channels.

\section{THE NEW TECHNIQUE FOR BASEBAND PULSE TRANSMISSION}

\subsection{Introduction}

Transmission of data may be either baseband or passband. Many commercial communication systems use baseband transmission directly such as T1 carrier systems, integrated services digital network (ISDN), coaxial cable and optical fiber local area networks (LANs), ultra-wideband (UWB) networks and radar applications [15], [16].

First, we define the rectangular pulse with an amplitude of $A$ and width of $\tau$ to be $A \operatorname{rect}(t / \tau))$. Assume that $x(t)$ is

$$
x(t)=A \operatorname{rect}(t / \tau)
$$

By using the definition in (1), the FT of $x(t)$ is

$$
X(f)=A \tau \operatorname{sinc}(f \tau)
$$

$x(t)$ and $X(f)$ areshown in Figure 1. The maximum value of $X(f)$ is $A \tau$ and occurs at $f=0$. The frequencies of zero crossings are $f=k / \tau$ where $k= \pm 1, \pm 2, \ldots, \infty$. The spectrum shows that the largest values occur at frequencies close to $f=0$ (main lobe) and other side lobes around it.

Then, the largest energy of the pulse spectrum exists in the main lobe (around 90\%) and the remaining energy is spread into the side lobes. So, the bandwidth $\Delta f$ of the pulse spectrum is the distance from $0 \mathrm{~Hz}$ to the first spectral null $(1 / \tau)$. At the receiver side, a low-pass filter (LPF) is designed with a bandwidth of $1 / \tau$ to extract the original pulse signal [17].

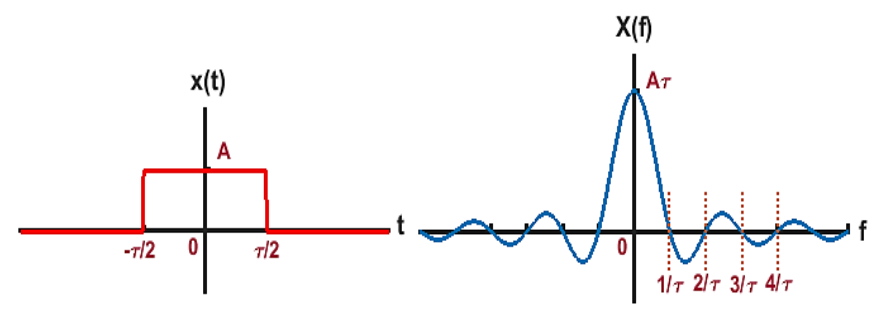

Figure 1: $x(t)$ and its Fourier Transform $X(f)$.

\subsection{The New Technique Concept}

Consider the suggested communication system (Transmitter and Receiver) shown in Figure 2 whereas a baseband pulse source transmitter is available. The baseband pulse signal will be assumed to be $f(t)$.

Generally, sending a baseband pulse depends on the pulse duration and the available channel bandwidth. If the channel bandwidth is smaller than the pulse spectral information main lobe, then a complete pulse distortion will occur, and the pulse information will be lost.

The presented new technique concept for baseband pulse transmission using a window from its spectrum is shown in Figure 3. The concept is based on taking the IFT of the given spectrum and then multiplying the distorted pulse by a pulse $p(t)$ of duration $\tau$, which is the same as the main pulse to be reconstructed.

The band range of the BPF can be freely selected to pass a spectral window taken from the pulse spectral information. This spectral window can be freely selected from a starting frequency $\omega_{s}$ to an end frequency $\omega_{e}$.

The selected window from the pulse spectral information will be sent through any band-limited channel that is available to pass the selected window from the baseband pulse. The transmitted window from the pulse spectral information will result to in a distorted pulse signal $g(t)$ and even lost information.

At the receiving side, the distorted pulse signal $g_{0}(t)$ will first pass through the new pulse reconstruction technique which is the main concept of this research. The new reconstruction technique will be fed with a priori information about the transmitted pulse duration.

The new baseband pulse reconstruction technique will be explained in the next section. 

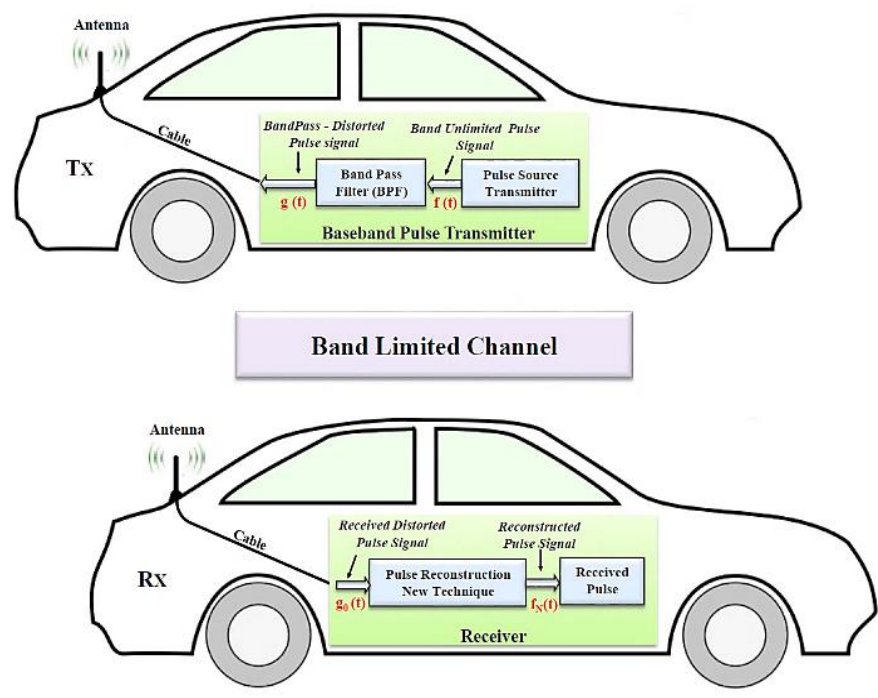

Figure 2: Baseband Pulse Transmission New Technique

\subsection{Baseband Pulse Reconstruction Technique}

Assume that the pulse source transmitter in Figure 2 transmits a pulse signal $f(t)$ as shown in Figure 4 (a). Its Fourier Transform $\mathrm{F}(f)$ is shown in Figure 4 (b). Assume that the transmitted window spectrum taken from the pulse is $G(f)$ as shown in Figure 4 (d). The spectral window frequency range extends from $f_{s}[\mathrm{~Hz}]$ to $f_{e}[\mathrm{~Hz}]$.

At the receiver side, the presented reconstruction technique goal is to determine the IFT $f(t)$ in terms of a finite segment $G(f)$ of $F(f)$ and then it extrapolates the original pulse spectrum making use of only the transmitted spectrum segment $G(f)$ and the a priori information about the temporal extent of the signal.

The received distorted pulse signal $g(t)$ due to the limited available spectrum is shown in Figure 4 (c).
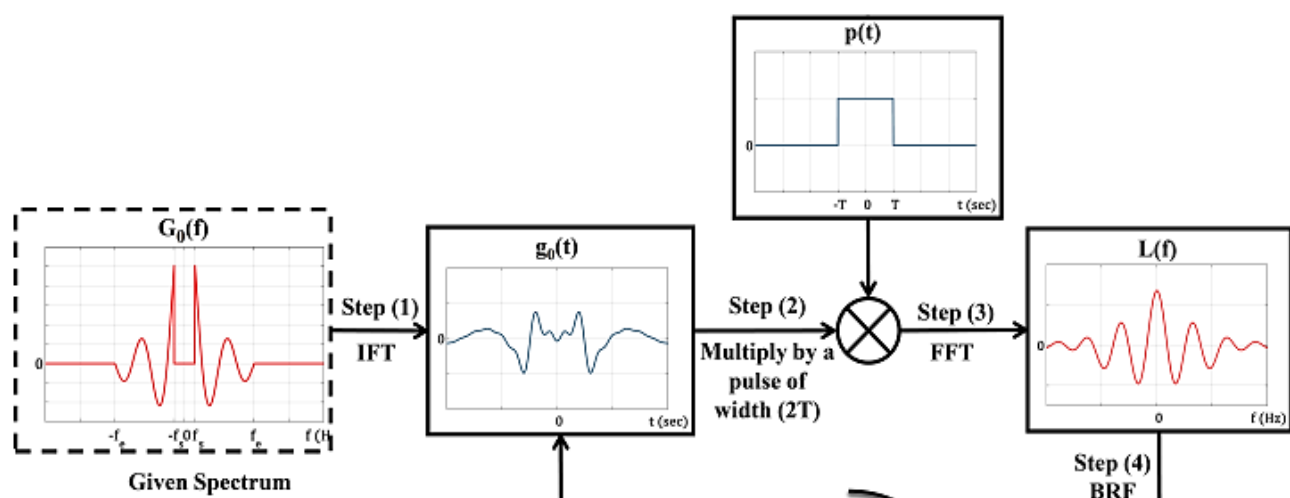

$\mathrm{G}_{0}(\mathbf{f})$
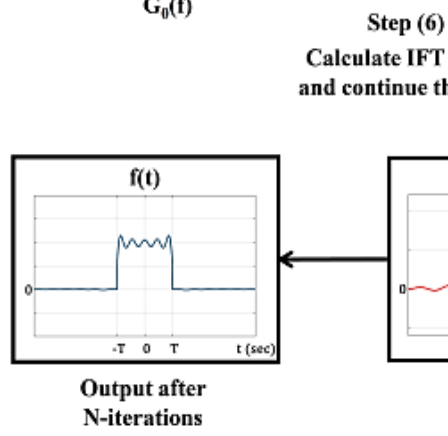

Calculate IFT again and continue the loop
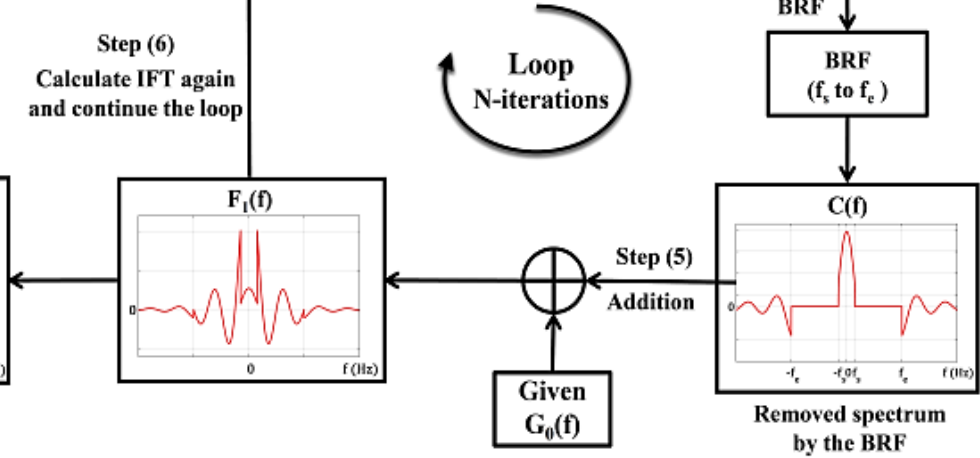

Figure 3: The New Technique concept and its software implementation.

Figure 3 illustrates the detailed steps of the pulse reconstruction technique, where the first step incorporates an IFT of the given baseband pulse selected band-pass spectrum $G_{o}(f)$, which results in the non-time limited signal $g_{0}(t)$.

The pulse reconstruction technique reinforces the time limiting operation by simply multiplying $g_{o}(t)$ by a gate function $\operatorname{rect}(t / \tau)$, where $\tau$ is the time extent of the signal to be reconstructed as shown in step 2. Step 3 is simply the FT back to the frequency domain, and as shown, the spectrum $L(f)$ is neither band limited nor equal to $G_{0}(f)$ within the interval window $\Omega=\left\{\omega: \omega_{s} \leq|\omega| \leq \omega_{e}\right\}$. The spectrum $L(f)$ is next applied to a band-reject-filter (BRF) with its pass-band extending from $f_{s}$ to $f_{e}$ as shown in step 4. This results in a spectrum $C(f)$.

Finally in step 5, the known portion of the signal, which is $G_{0}(f)$, is inserted in the dead spectrum space $C(f)$, giving rise to the first spectrum estimate $F_{1}(f)$ which is principally a discontinuous function and hence doesn't represent a timelimited signal. 
Ahmed F. Ashour et al., International Journal of Advanced Trends in Computer Science and Engineering, 9(4), July - August 2020, 5222 - 5228

The reconstruction technique will re-impose the criterion of time limitation again as before, which is the beginning of the next iteration. We will denote the results of $\mathrm{N}^{\text {th }}$ iteration by $F_{N}(f) \leftrightarrow f_{n}(t)$.

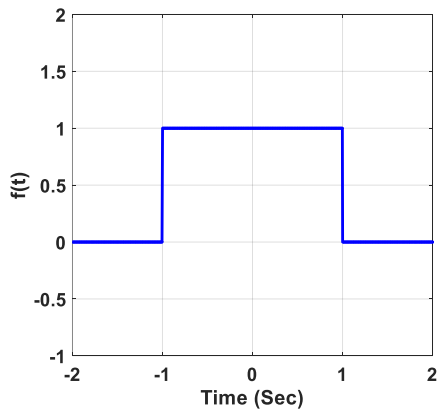

(a)

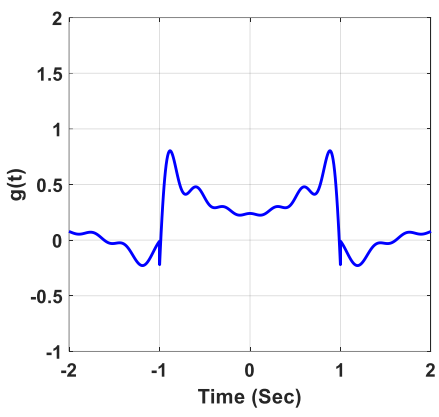

(c)

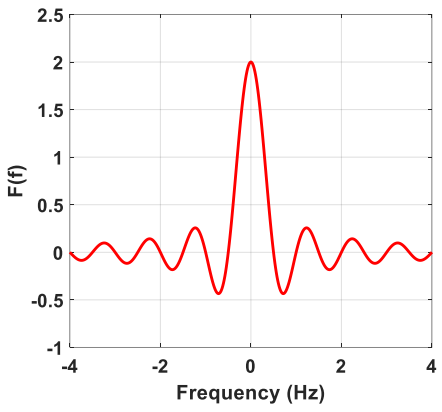

(b)

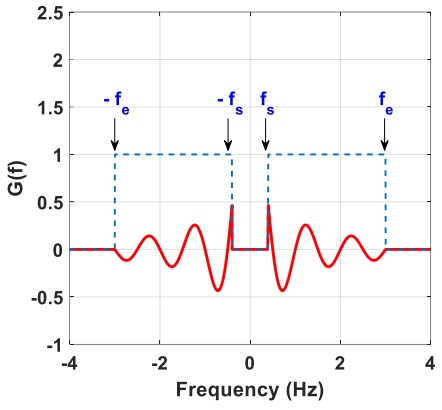

(d)
Figure 4: Baseband Pulse Spectrum Reconstruction Problem (a) The original pulse signal (b) FT of the original pulse signal (c) Transmitted distorted pulse signal (d) Transmitted window spectrum.

\subsection{Mathematical Modeling of Baseband Pulse Recon- struction New Algorithm}

From Figure 3, steps 1, 2, and 3 are simply a duration limiting operation, so let the duration limiting operator $D_{T}$ be defined as

$$
D_{T}\{h(t)\}=h(t) \operatorname{rect}\left(\frac{t}{\tau}\right)
$$

where $\operatorname{rect}(t / \tau)$ is a gate function adjusted with a durartion equal to the time extent of the time limited signal to be reconstructed, and $\tau / 2$ is the positive time extent of that signal.

The given segment of the spectrum can be considered as an output of a system composed of two low pass filters connected as shown in Figure 5, where the end frequency $\omega_{e}>\omega_{s}$

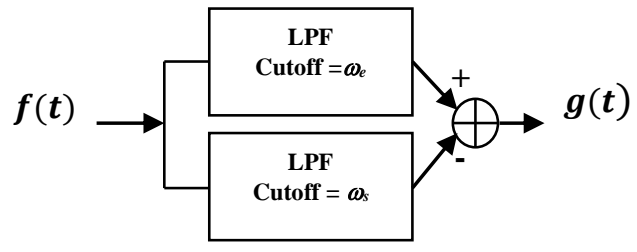

Figure 5: Modeling of the band pass signal $g(t)$
An LPF operator is defined to be $B_{\omega x}\{h(t)\}$ where

$$
B_{\omega x}\{h(t)\}=h(t) * \frac{\omega_{x}}{\pi} \operatorname{sinc}\left(\frac{\omega_{x}}{\pi} t\right)
$$

Now, the operation of rejecting the band starting from $f_{s}$ to $f_{e}$ by using the BRF in step 4 , can be modeled by the band reject operator $B_{B R F}$ where

$$
B_{B R F}\{h(t)\}=\left[B_{\omega s}+\left(1-B_{\omega e}\right)\right] h(t)
$$

where $\omega_{s}$ and $\omega_{e}$ are the start and end measuring frequency of $G(\omega)$.

With the above described notations, assume that the time limited function to be reconstructed is $f(t)$ with positive time extent $\tau / 2$, and by making use of the identity

$$
D_{T}\{f(t)\}=f(t)
$$

This follows from the time limitedness of $f(t)$. Define $g(t)$ to be the given inverse Fourier transformation of the given spectrum $G(\omega)$, so $g(t)$ can be described by

$$
g(t)=B_{\omega e}\{f(t)\}-B_{\omega s}\{f(t)\}
$$

Equation (12), can be re-written in the form

$$
g(t)=\left[1-1+\left(B_{\omega e}-B_{\omega s}\right) D_{T}\right] f(t)
$$

If the operator in square brackets can be inverted, then we have

$$
f(t)=\left[1-\left(1-\left(B_{\omega e}-B_{\omega s}\right) D_{T}\right]^{(-1)} g(t)\right.
$$

For inversion, we can generalize the geometric series and writing (14) as follows

$$
f(t)=\sum_{n=0}^{\infty}\left[1-\left(B_{\omega e}-B_{\omega s}\right) D_{T}\right]^{n} g(t)
$$

From which we have:

$$
f_{N}(t)=\sum_{n=0}^{N}\left[1-\left(B_{\omega e}-B_{\omega s}\right) D_{T}\right]^{n} g(t)
$$

Equation (16) can be rearranged to obtain our Algorithm

$$
f_{N+1}(t)=g(t)+\left[1-\left(B_{\omega e}-B_{\omega s}\right) D_{T}\right] f_{N}(t)
$$

with initialization

$$
f_{0}(t)=g(t)
$$

In the following section, we will introduce a numerical validation example for the baseband pulse reconstruction from its selected band pass spectral information window.

This will prove that the baseband pulse can be transmitted over any band-limited communication channel. 


\section{RESULTS AND DISCUSSION}

\subsection{Baseband Pulse Reconstruction Numerical Validation}

Consider the transmitter and receiver system shown in Figure 2. Let the transmitted baseband pulse signal be a rectangular function as in (6). Then, the baseband pulse signal will be modeled as

$$
f(t)=\operatorname{rect}(t / 2)
$$

where the amplitude $A=1$ and pulse duration $\tau=2 \mathrm{sec}$. The FT associated of this pulse can be easily evaluated using (7) to be

$$
F(f)=2 \operatorname{sinc}(2 f)
$$

Consider Figure 4 and assume that the given pulse duration is equal to $\tau=2[\mathrm{sec}]$. Assume that the transmitted bandpass spectrum $G(f)$ from the pulse spectrum is known between the starting frequency $f_{s}=0.3[\mathrm{~Hz}]$ to $f_{e}=3[\mathrm{~Hz}]$. It was clear that the associated IFT, which is $g(t)$ is a severely a distorted version of the main function $f(t)$ as shown in Figure 3.

This is due to the small finite sent segment of $f(t)$. After receiving $G(f)$, shown in Figure 4 (d), the receiver will apply the presented new baseband pulse reconstruction technique shown in Figure 3 which is explained in section II.

The presented technique will be fed with a priori information about the pulse duration (here $\tau=2$ [sec.]) and will be run for a number of $\mathbf{N}$-iterations.

Firstly, we divided the main lobe of $F(f)$ to start from starting frequency $f_{s}=0[\mathrm{~Hz}]$ up to an end frequency $f_{e}=0.5[\mathrm{~Hz}]$ of the pulse's spectrum as shown in Figure 4 (b) into four quadrants.

Figure 6 shows different transmitted signals after windowing the main lobe of the original pulse spectrum. Figure 7 shows the reconstructed spectrum $g(t)$ after $\mathrm{N}=1,5,10,50$ and 500 iterations and the corresponding pulse signal in time domain.

By increasing the number of iterations to $\mathbf{N}=1000$, the baseband pulse spectrum is completely reconstructed and consequently the baseband clean pulse signal is reconstructed with the well-known Gibbs effect [2].

Further constructed Spectrum, it is clear that the spectrum is approximately similar to the original $F(f)$ (Figure 4), so that the time domain pulse is approximately reconstructed well at the receiver side. This means that the baseband pulse signal can be sent over a bandlimited channel by freely selecting a spectral window from its baseband spectrum.

Figure 8 shows a summary of the reconstructed pulse with iterations from $\mathrm{N}=1$ up to $\mathrm{N}=500$.
Figure 9 shows the new technique's energy efficiency ( $\left.\mathrm{E}_{\text {Received }} / \mathrm{E}_{\text {Transmitted }}\right) \%$ for different windows of transmitted spectrum.
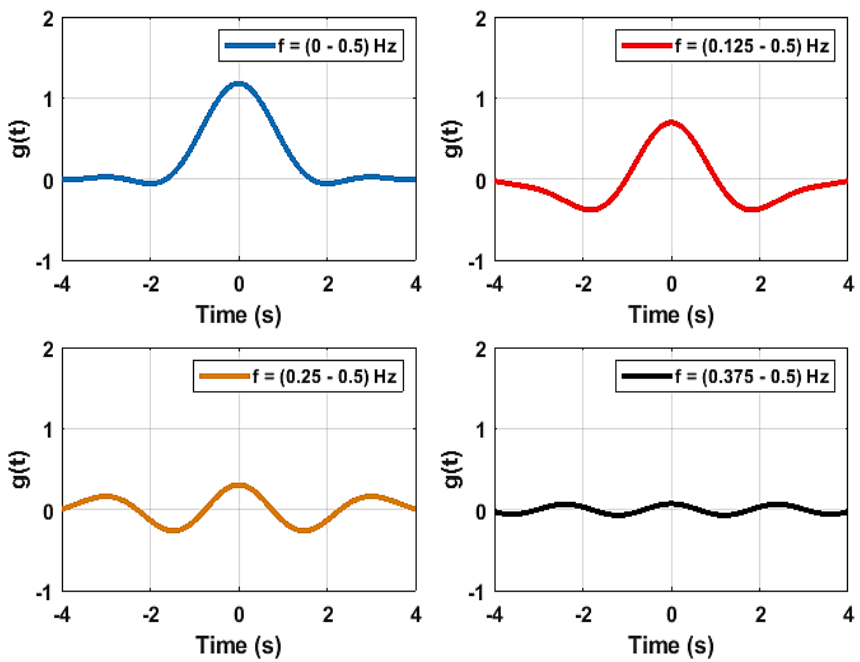

Figure 6: Transmitted signals for different spectrum windows

The efficiency is measured when sending four different windows of the main lobe spectrum and the algorithm is run for 5000 iterations. At $N=1$, the efficiency of the new technique is around $90 \%$ which is the same as the conventional method of filtering the main lobe.

By increasing $N$ to be 30 iterations for the same window, more than $94 \%$ of the signal's energy can be received. By decreasing the window to $(0.125-0.5)[\mathrm{Hz}]$, around 100 iterations are needed to receive the same energy of the main lobe window.

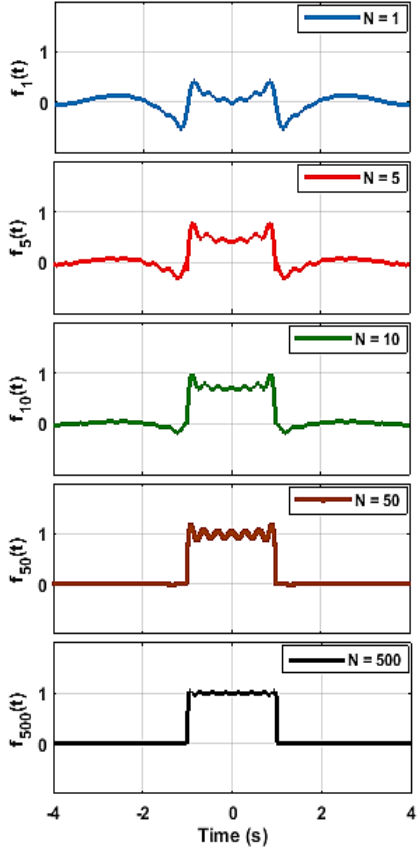

(a)

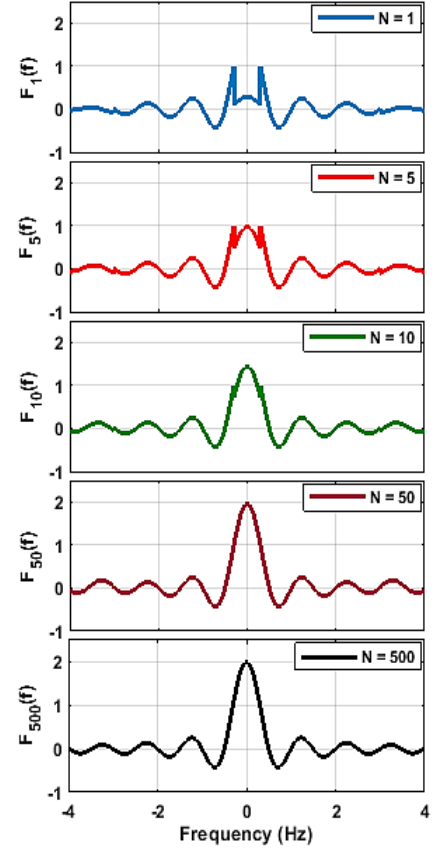

(b)
Figure 7: (a) Reconstructed baseband pulse for different iterations (b) Spectrum of the reconstructed baseband pulse in part (a) 
Ahmed F. Ashour et al., International Journal of Advanced Trends in Computer Science and Engineering, 9(4), July - August 2020, 5222 - 5228

If we decrease the window to be $(0.25-0.5)[\mathrm{Hz}]$, around $93 \%$ of the signal's energy can be recovered for 500 iterations.

If we decrease the window to be $(0.375-0.5)[\mathrm{Hz}]$, around $92 \%$ of the system's energy may be received for 1000 iterations and increased for more signal energy recovery. Thus, saving $75 \%$ of signal's bandwidth compared to conventional method.

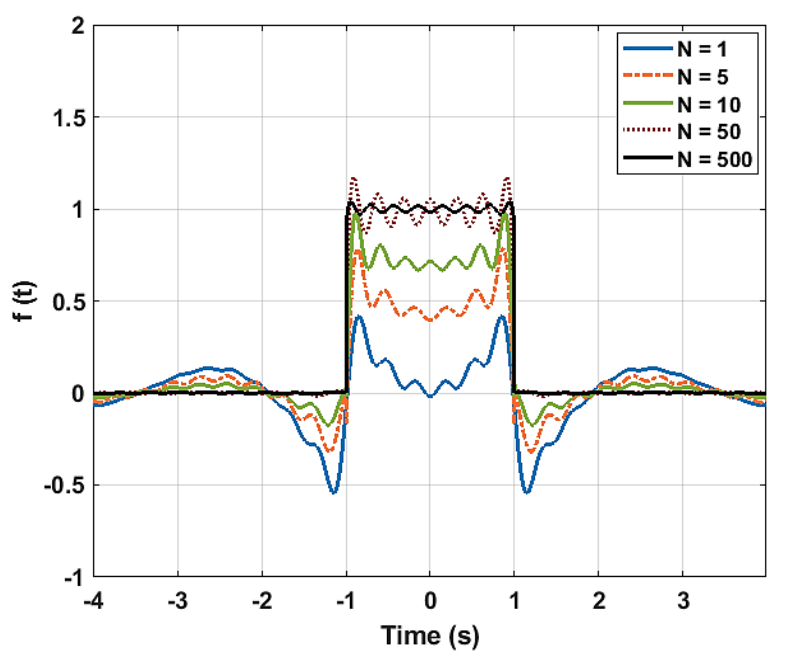

Figure 8: The Reconstructed pulse $f(t)$ for different iterations.

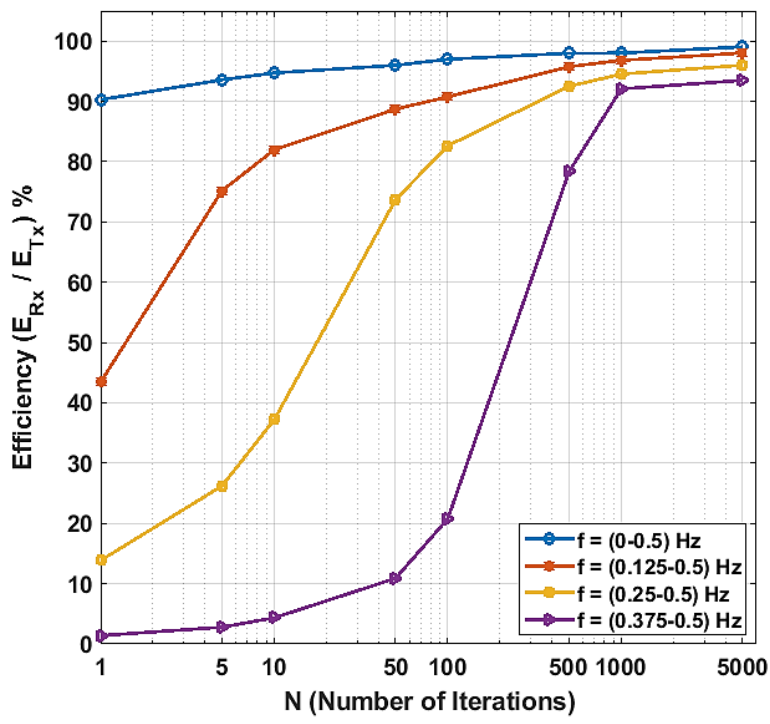

Figure 9: The efficiency of the new algorithm for different transmitted spectrum windows and different iterations.

\section{CONCLUSION}

We proposed a new concept for baseband pulse transmission over a bandlimited channel. The concept is based on selecting any band-pass window from the baseband pulse main spectrum. The selected pulse spectral window should pass through the given channel bandwidth. At the receiving side, a new algorithm based on band reject filtering is used for reconstructing the baseband pulse given only a segment of its spectrum. This segment represents the measurement done on the spectrum of a time limited pulse function $f(t)$ from a starting measuring frequency $f_{s}$ up to an end frequency $f_{e}$. It should be noted that as the starting measuring frequency increases, due to limitation of measuring devices for example, the only effect on our proposed algorithm is the increase in number of iterations.

Therefore, we will be still be able to reconstruct the original spectrum (at the receiver side) of the signal, thus the time limited baseband pulse function can be easily reconstructed. The new presented concept and technique for baseband pulse transmission should find applications for Mobile Communications and microwave measurements with instrumental limitations.

\section{REFERENCES}

1. Nahvi, Mahmood. Signals \& Systems. McGraw-Hill Higher Education, 2014.

2. Kulkarni, A. \& Rani, Elizabeth. (2019). Unconstraint 2level cost optimization coding for signal processing. International Journal of Advanced Trends in Computer Science and Engineering. 8.

3. Omar, Abbas. Band-Limited Reconstructions in Microwave Imaging. In 2019 IEEE MTT-S International Conference on Numerical Electromagnetic and Multiphysics Modeling and Optimization (NEMO), pp. 1-4. IEEE, 2019.

4. Brugnoli, Emanuele, Elena Toscano, and Calogero Vetro. Iterative reconstruction of signals on graph. IEEE Signal Processing Letters (2019).

5. Indira, N.Durga. (2020). Compressive Sensing For Speech Signals. International Journal of Advanced Trends in Computer Science and Engineering. 9. 11471150. 10.30534/ijatcse/2020/38922020.

6. Zhang, Qun, Yijun Chen, Yongan Chen, Long Chi, and Yong $\mathrm{Wu}$. A cognitive signals reconstruction algorithm based on compressed sensing. In 2015 IEEE 5th Asia-Pacific Conference on Synthetic Aperture Radar (APSAR), pp. 724-727. IEEE, 2015.

7. Huang, Honglin, and Anamitra Makur. A new iterative reconstruction scheme for signal reconstruction. In APCCAS 2008-2008 IEEE Asia Pacific Conference on Circuits and Systems, pp. 336-339. IEEE, 2008.

8. Nakajima, Nobuharu. Ultrashort pulse reconstruction using a deterministic phase retrieval method with Gaussian-envelope gates. Optical Review 26, no. 1 (2019): 77-84.

9. Sun, Heng, Hongjun Liu, Qibing Sun, Nan Huang, Zhaolu Wang, and Jing Han. Numerical analysis of pulse signal restoration by stochastic resonance in a buckled microcavity. Applied optics 55, no. 12 (2016): 3351-3355. 
10. Zhou, Wei, Wei Xie, Xuesong Cao, Chao Wu, and Shiping $\mathrm{Zhu}$. Iterative adaptive spectrum reconstruction algorithm for MIMO SAR. In 2016 IEEE 13th International Conference on Signal Processing (ICSP), pp. 187-191. IEEE, 2016.

11. Buckingham, M. J., and E. A. Faulkner. The principles of pulse signal recovery from gravitational antennas. Radio and Electronic Engineer 42, no. 4 (1972): 163 171.

12. Milman, Mario. Extrapolation and optimal decompositions: with applications to analysis. Springer, 2006.

13. Zhao, Hui, Ruyan Wang, Daiping Song, and Dapeng Wu. An Extrapolation Algorithm for $\$(\mathbf{a}, \mathbf{b}, \mathbf{c}, \mathbf{d}) \mathbf{\$ -}$ Bandlimited Signals. IEEE Signal Processing Letters 18, no. 12 (2011): 745-748.

14. Xing-Wie Zhou, Xiang-Gen Xia, The Extrapolation of High-Dimensional Band-Limited Functions, IEEE Trans. Acoustics, Speech, and Signal Processing , vol.37, No.10, pp.1576-1580, October 1989.

15. Gitlin, Richard D., Jeremiah F. Hayes, and Stephen B. Weinstein. Data communications principles. Springer Science \& Business Media, 2012.

16. Pahlavan, Kaveh, and Prashant Krishnamurthy. Principles of wireless access and localization. John Wiley \& Sons, 2013.

17. Sahley, Tony L., and Frank E. Musiek. Basic Fundamentals in Hearing Science. Plural Publishing, 2015. 